\title{
EMBEDDED MANAGEMENT SYSTEM FOR OUT PATIENT DEPARTMENT
}

\author{
C. Kavitha ${ }^{1}$ A. Venkat Ramana ${ }^{2}$ S. Sushma Raj ${ }^{3}$ \\ ${ }^{1}$ Associate Professor, Department of Electronics/ Physics, GITAM Institute of Science, \\ GITAM University, Visakhapatnam-530 045, 9885058061, \\ kavithachandu2000@yahoo.co.in \\ ${ }^{2}$ Technical Lead, Gain Span India Pvt. Ltd. Bangalore. \\ Venkat. Ramana@Gainspan. com \\ ${ }^{3}$ Assistant Professor, GITAM Institute of Science, \\ GITAM University, Visakhapatnam-530 045, \\ s.sushmarajegmail.com
}

\begin{abstract}
In the resent past one field which has brought in many changes in the human lives is the technology. Technology is meant for bringing innovation in human lives and reduces the efforts to accomplish a task. Corporate hospitals today employ large number of doctors specialized in various fields. Out patients who wish to consult a particular doctor finds it very difficult to locate their respective doctor's cabin. Keeping the patient's difficulty of tracking the doctors, an embedded based device has been designed to assist patients to easily locate the doctor's cabin. The device displays the patient's name and token number outside of the consultant's room
\end{abstract}

\section{KEYWORDS}

RS-485, LCD, Memory Card, Key board, RTC.

\section{INTRODUCTION}

In life daily, an embedded system plays a vital role in making life style easy and uncomplicated. An embedded system is defined as "A Programmable device, which can be designed to perform dedicated functionalities of a trivial system to scheduled functionalities of a large system".

The main aim of this project is to reduce the efforts of the patients who come to the hospital to consult the doctor to resolve their health concerns. This project is accomplished using regular embedded devices like Microcontrollers, SD card, LCD, RS-485 and using SPI(Serial Peripheral Interface) communication protocol to establish communication between the keyboard and the Microcontroller.

\section{BLOCK DIAGRAM}

The block diagram of outpatient unit is shown in Figure 1. Figure 2 depicts OPD(Out Patient Department) unit in different consultant's room and Figure 3 depicts the display unit which displays the patient name and token number outside the consultant's room.

DOI : 10.5121/ijesa.2012.2305 
International Journal of Embedded Systems and Applications (IJESA) Vol.2, No.3, September 2012

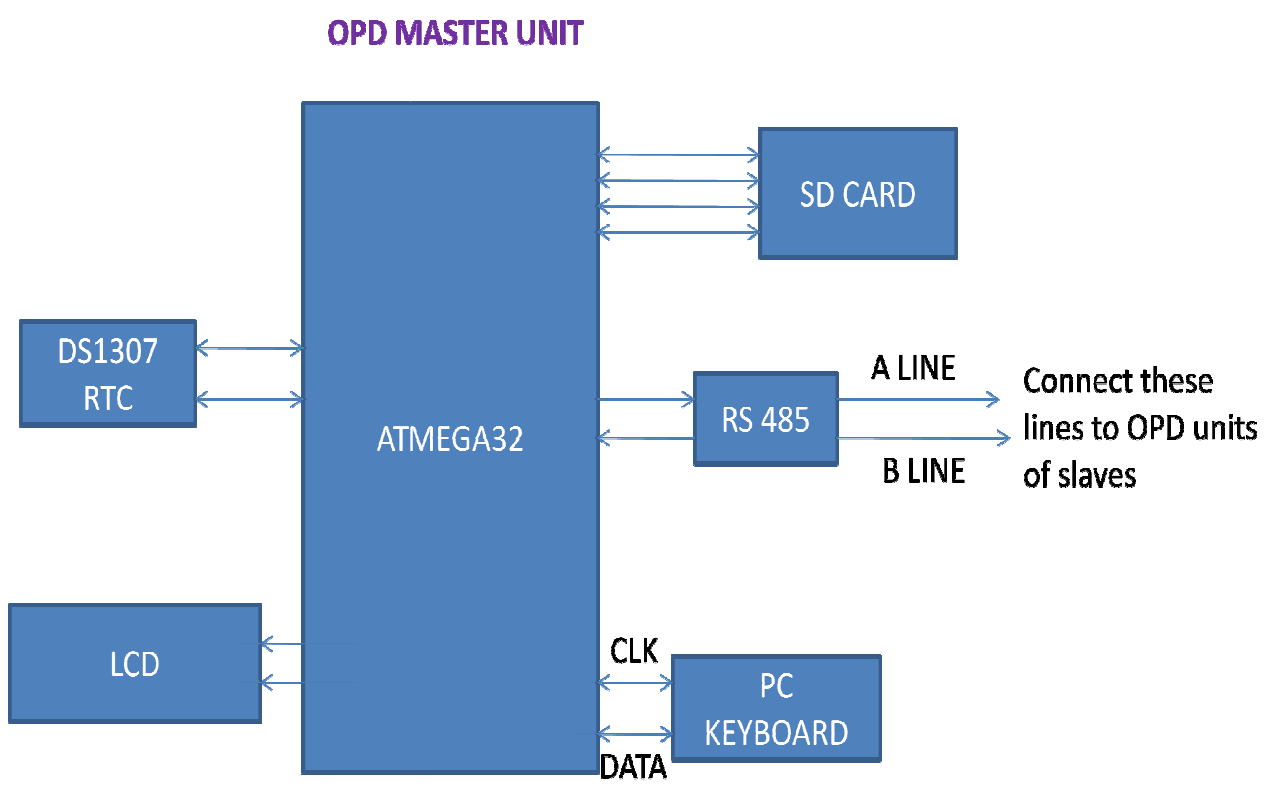

Figure 1: Master OPD unit

OPD UNIT 1

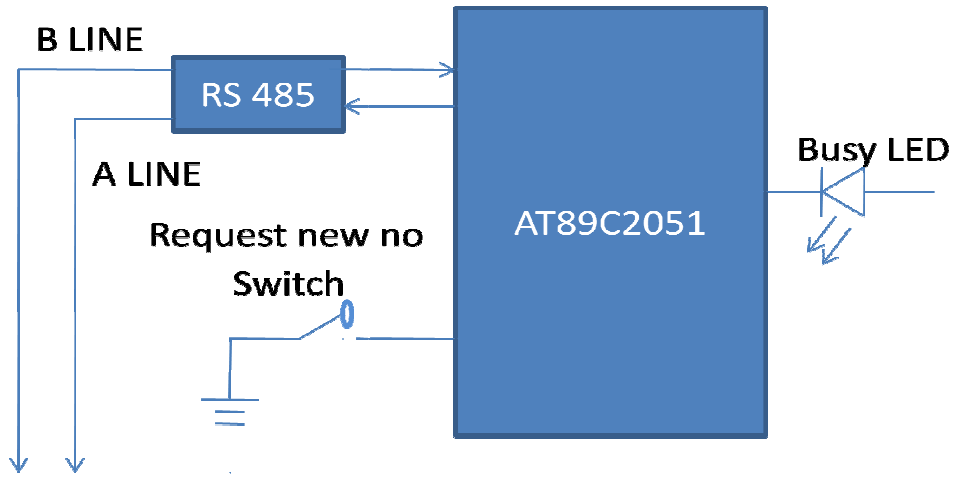

Connect these lines to

main controller $A \& B$

lines(RS 485)

And also connect these lines to different OPD units of $A \& B$

Figure 2: OPD slave unit 
International Journal of Embedded Systems and Applications (IJESA) Vol.2, No.3, September 2012

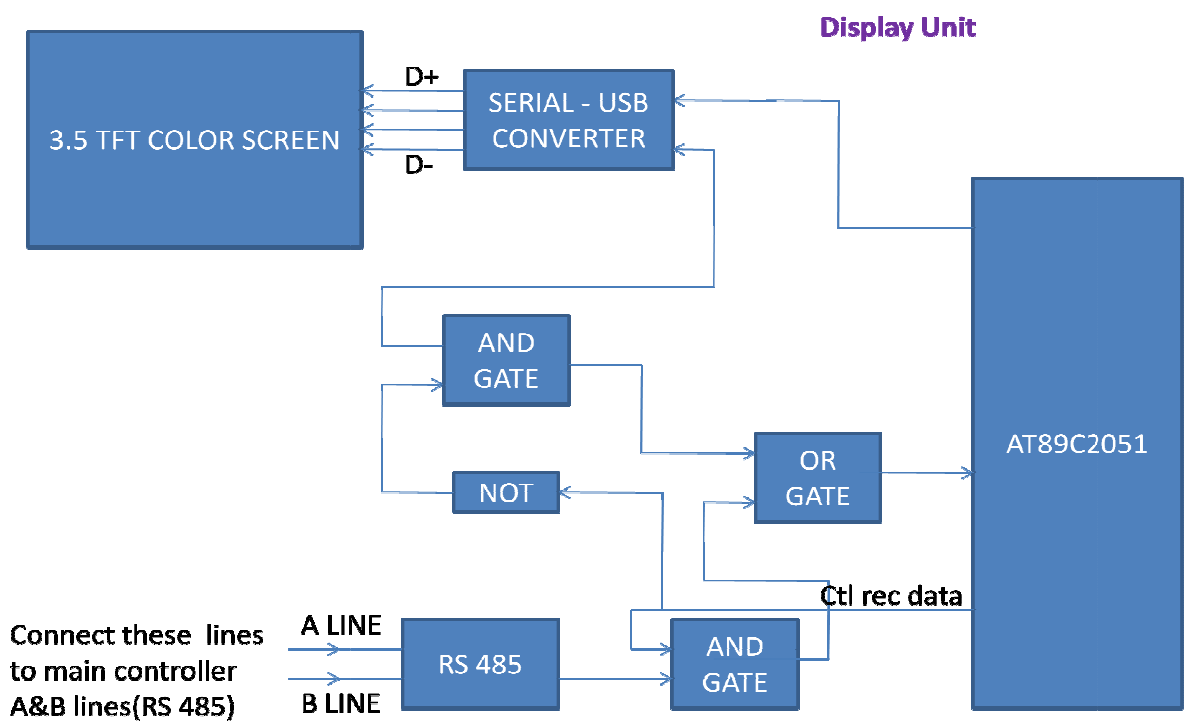

TFT Display controller - used to display patient

Name and Token number

Figure 3: Display unit system

\section{HARDWARE SCHEMATIC}

The hardware schematics of Microcontroller based OPD system shown in schematic 1, 2, 3, 4, 5 and 6.

\subsection{Master OPD Unit System}

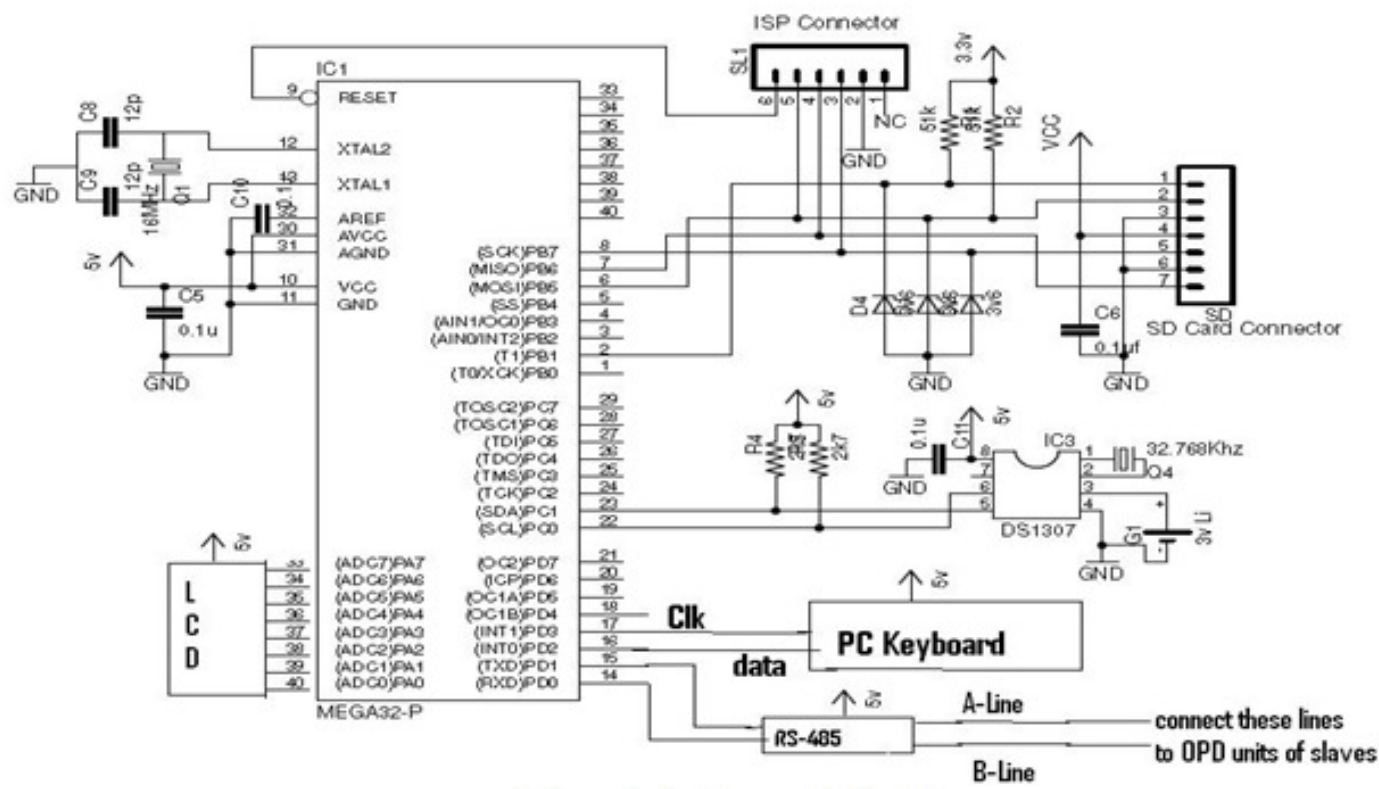

Schematic 1: Master OPD unit|

Schematic 1: Master OPD unit 
International Journal of Embedded Systems and Applications (IJESA) Vol.2, No.3, September 2012

\subsection{OPD Slave 1}

OPD slave 1 is placed inside the consultant's room to inform Master OPD unit to send the next token number of corresponding consultant.

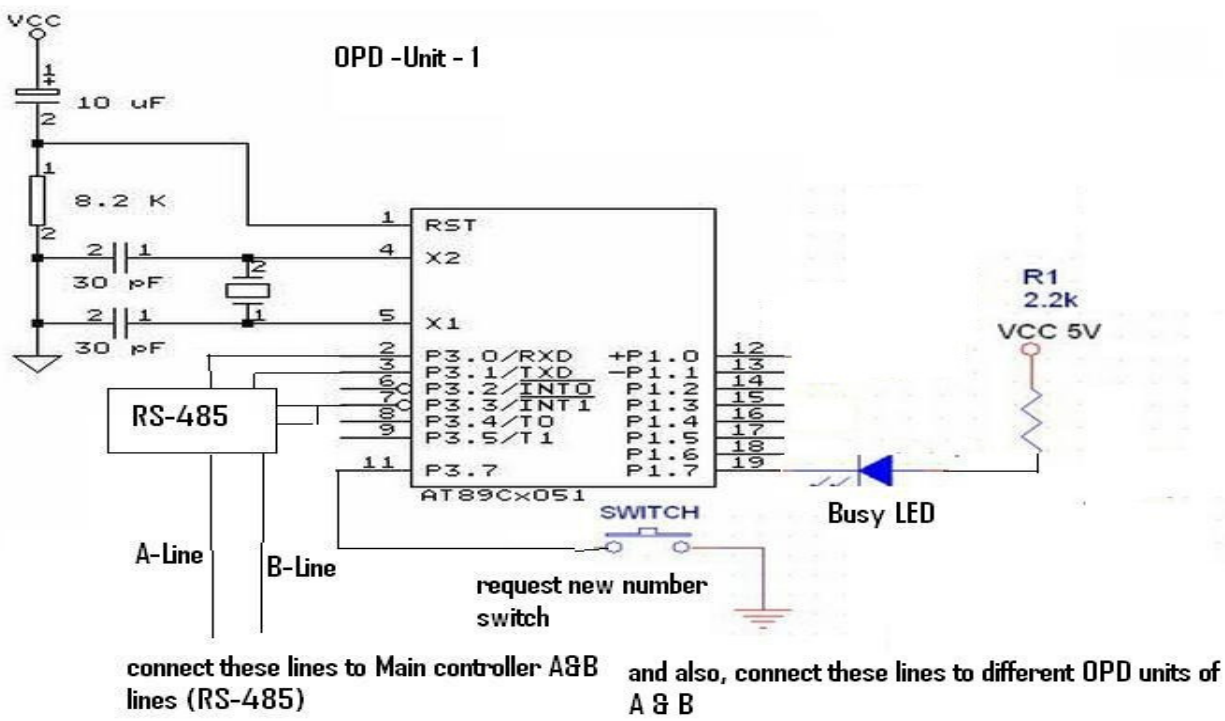

Schematic 2: Slave OPD unit 1

\subsection{OPD Slave 2}

OPD slave 2 is placed inside the consultant's room to inform Master OPD unit to send the next token number of corresponding consultant.

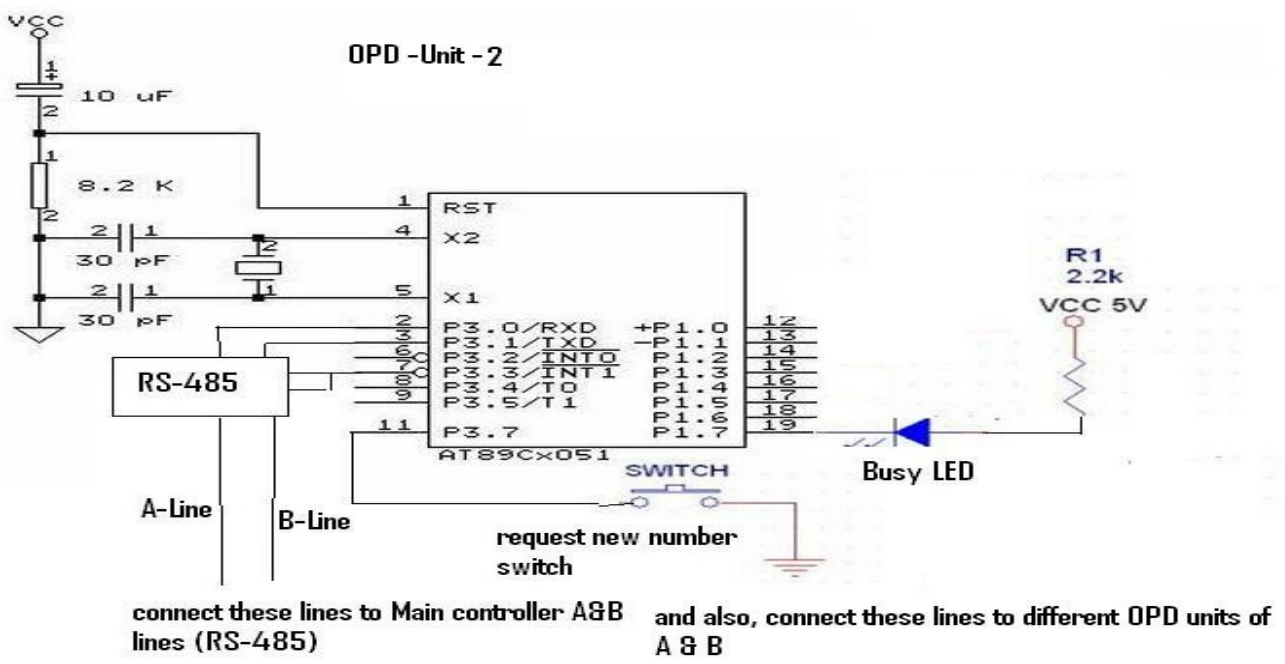

Schematic 3: Slave OPD unit 2 


\subsection{OPD Slave 3}

OPD slave 3 is placed inside the consultant's room to inform Master OPD unit to send the next token number of corresponding consultant.

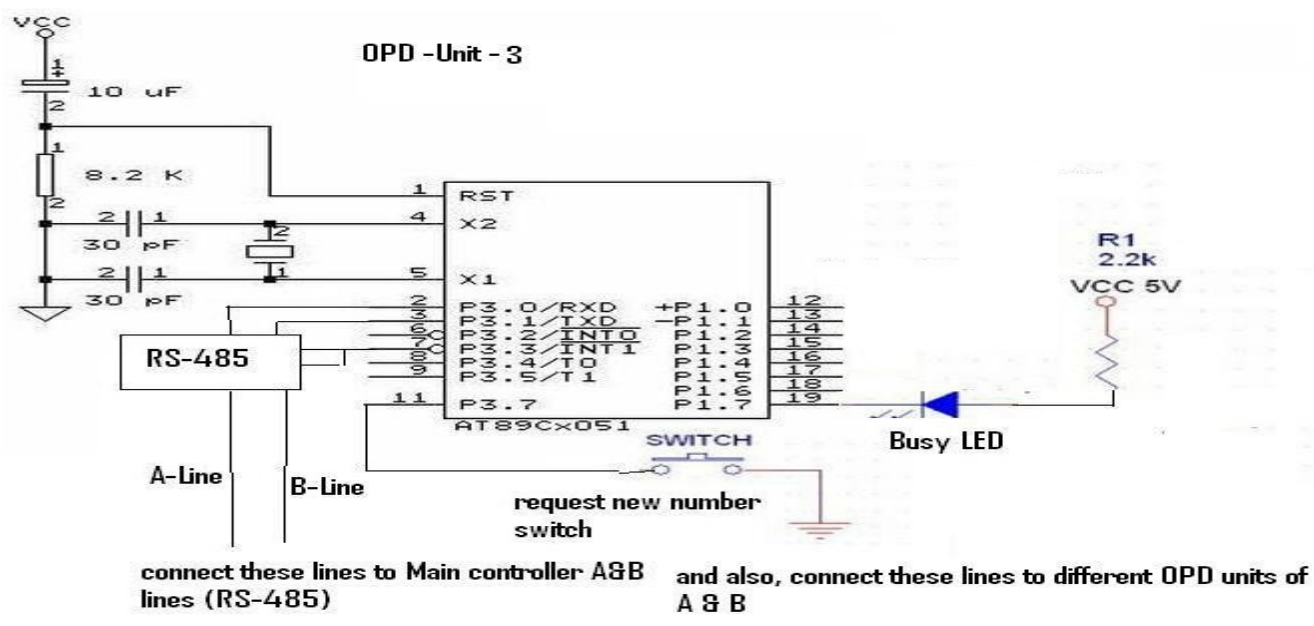

Schematic 4: Slave OPD unit 3

\subsection{Display Unit}

ATFT screen placed outside each consultant's rooms, for each patient to know about their token number and name which displayed on TFT (Thin Film Transistor)display.

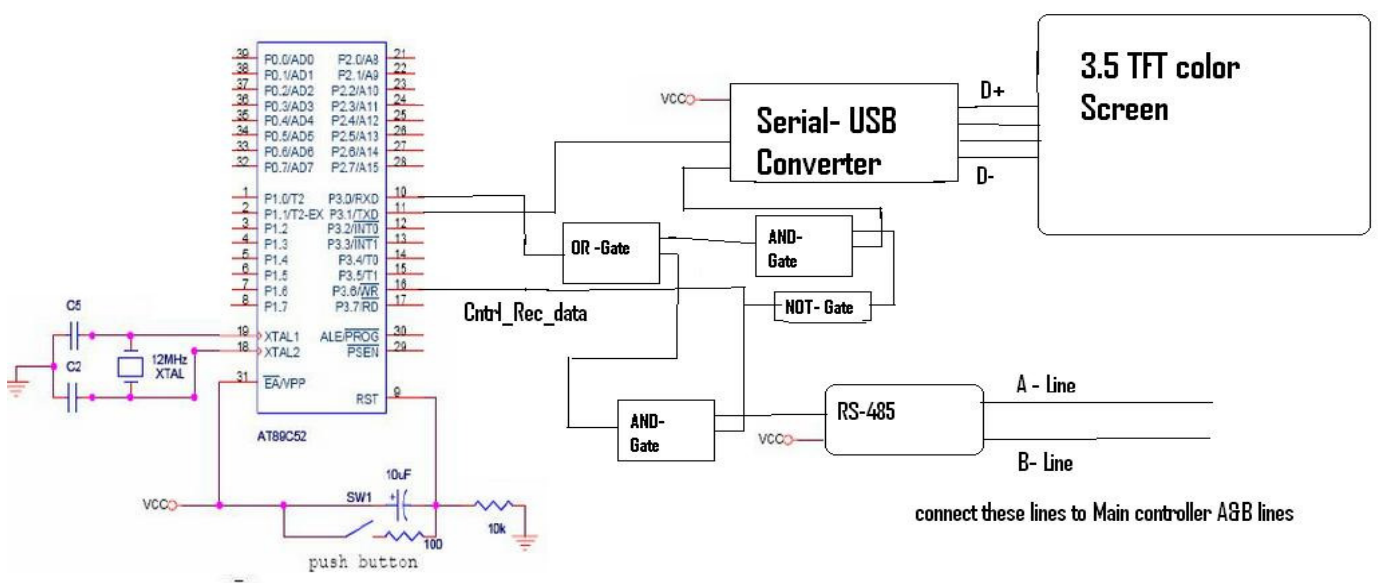

TF display controller - used to display patient number, Name \& Doctor specialist

Schematic 5: Display unit system

\subsection{Power Supply Unit}

Regulated 5VDC power supply is required for Microcontrollers and regulated 3.3VDC is required for SD Card interfacing. 


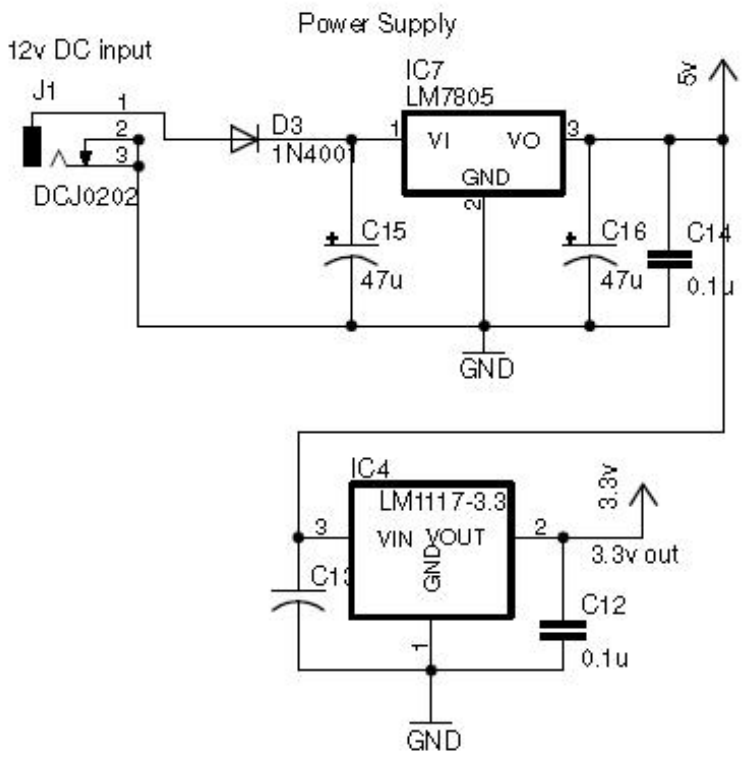

Schematic 6: Power supply unit

\section{DESCRIPTION}

The Main micro controller (Master OPD Unit) is interfaced with the several commonly used devices such as RTC(Real Time Clock), Memory Card, LCD and PC Key Board. Each of these devices have their unique usage in this process. The RTC is used to store the Event Date and Time, and I2C communication is used to transfer the data between the main microcontroller unit and RTC. PC keyboard is used to record the details of the patient in the Memory card and the communication between these two is done using SPI interface. The Memory card is used to store the details of the patients for the purpose of displaying on the TFT screen which is deployed outside the every doctor's room.

Depending on the number of departments in the hospital, equal number of TFT screens are used to list down the patient's names who have got themselves registered under each doctor. Each doctor's room consists of an OPD slave unit, used for making a communication between the reception (Master OPD Unit) and their individual cubicles, this requirement can be captured using RS-485 communication protocol which is similar to serial communication protocol but differs in the distance over which RS-485 transmits the data. Each OPD slave contains one slave microcontroller, switch and LED. The slave microcontroller is used to get the data from the main microcontroller (Master OPD unit) to proceed further. The switch is interfaced with the slave microcontroller unit to start the request procedure i.e. when the switch is pressed the OPD slave microcontroller sends request to main microcontroller (Master OPD Unit) requesting for the next patient's details; as soon as the main microcontroller gets notified, it gets the next patient's data from the Memory card using SPI communication protocol. The LED usually abbreviated as Busy LED indicates the status of the request which is made by a doctor. Initially the LED begins in an OFF condition. After the treatment is done, the doctor presses the switch to notify the main microcontroller unit, then the LED is tuned to active (ON) State; when the OPD slave gets the data from the MCU, the OPD slave LED automatically turns off. The same information can be used to display on the TFT screen which is interfaced with the OPD main microcontroller unit using RS-485. The TFT screen is the main feature of this technology; it displays the list of the patients who have got registered under each doctor. 
International Journal of Embedded Systems and Applications (IJESA) Vol.2, No.3, September 2012

\section{HARDWARE COMPONENTS}

\subsection{Liquid Control Display}

It is used at the reception to let the receptionist know about the number of people registered. It also serves the purpose of ensuring whether the receptionist is storing the details in the correct pattern or not.

\subsection{MAX-485}

It is the important component in this technology since it can be used to establish the RS-485 communication; establishing this communication is so important because it transfers the data from the main microcontroller to the slave microcontroller unit.

\subsection{Key Board}

It is very common and useful embedded device and can be used to store the details in the memory card and this stored data can be used to display on the LCD.

\subsection{Memory Card:}

It is a very basic electronic device which we use frequently in our daily life. This is used for storing the Data which is very useful in displaying on the LCD, which is the main essential feature of this technology.

\subsection{RTC}

It is a very basic embedded device which stores the date and time of the events happening on daily basis, and the communication between micro controller and RTC is established using I2C communication protocol.

\subsection{TFT screen}

It is a very vital component of this technology which is used to display the names of the patients who are registered. 
International Journal of Embedded Systems and Applications (IJESA) Vol.2, No.3, September 2012

\section{FLOW CHART}

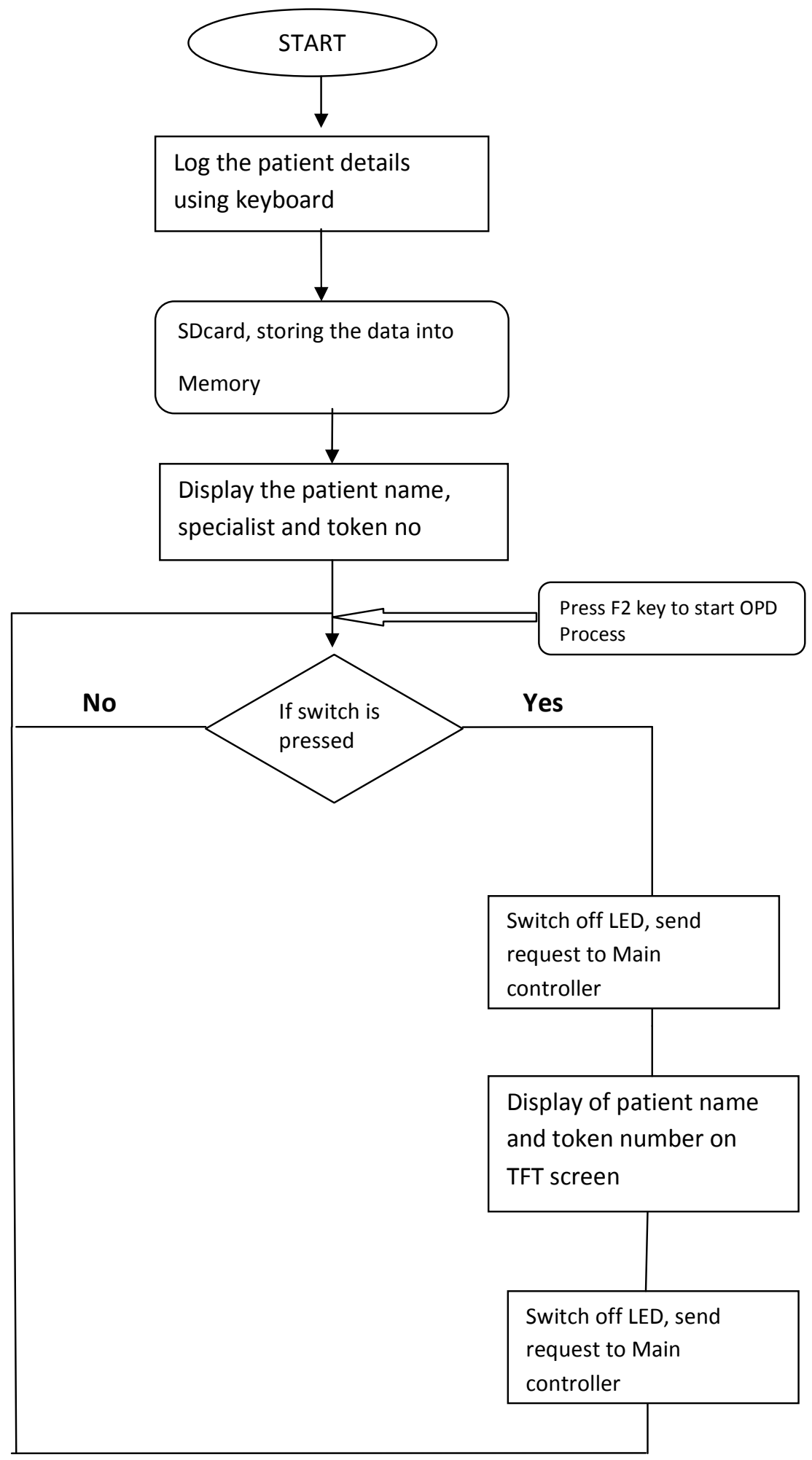


International Journal of Embedded Systems and Applications (IJESA) Vol.2, No.3, September 2012

\section{PROCEDURE}

The Main micro controller is interfaced with the RTC, PC keyboard and Memory Card. The PC Keyboard is interfaced with the Main Microcontroller Unit with a view to make a note of the details of the person who comes to the hospital and the same is stored in the memory card. Details include the person's name, disease, token number and the name of the doctor the patient wishes to consult. The RTC is accomplished with the main microcontroller with an intention of storing the event date and time, So that details can be used further. The communication between micro controller and RTC is e accomplished using I2C communication protocol. The TFT color screen displays the patient's name and token which is received (using RS-485 communication) from the main microcontroller unit. Once the doctors get into their individual cubicles, the receptionist presses the start button which is nothing but a key (F2) from the PC keyboard. The start button is dedicated for beginning the functionality of this concept. As soon as the start button (F2 Key) is pressed the names of the registered patients under each doctor is displayed on the TFT color screen which is deployed outside the doctor's room.

The TFT screens are interfaced using serial- USB cable. In the doctor's room one more micro controller unit (OPD Slave) is deployed which is usually interfaced with the Switch to send the request to the main microcontroller to get the information about the next patient. In Each Doctor's room, one LED is deployed to indicate the status of the request made by the doctor using a switch. This LED is abbreviated as Busy LED. At the beginning, Busy LED starts in switch off condition; once the Doctor is done with the treatment for one particular patient, the doctor presses the switch, as a result the LED is tuned to active state. This request alerts the main microcontroller to send the details of the next person listed in the Memory Card. Once the Main Microcontroller is done with the Data fetching from the Memory card using SPI communication protocol, the Microcontroller transfers the data using RS-485 to the slave microcontroller. Once the slave microcontroller receives the data, the Busy LED is tuned to put off state indicating that the request made by the doctor is processed. The whole communication between the Main microcontroller unit and the slave microcontroller is deployed in the doctor's room using RS-485 communication. At the reception also, the LCD is deployed to get rid of the numbers which are being stored in the Memory Card.

\section{PRECONDITIONS}

To make this product up and running following are the prerequisites which have to be followed.

i. Make sure that the Memory card is interfaced with the Main Microcontroller unit properly.

ii. Check to ensure that the Max-485 is active so that proper Communication can be enabled.

iii. Make sure that the LCD is initialized before using it for the displaying purpose

iv. Check for the correctness of the interface which has been made between the Microcontroller unit and the Keyboard, such that the details of the patient can be sent to the Memory card via Microcontroller properly. 
International Journal of Embedded Systems and Applications (IJESA) Vol.2, No.3, September 2012

\section{RESULTS}

The concept of the "Outpatient Department" has been designed and developed. It can be upgraded to wireless technology for future purpose.

\section{CONCLUSIONS}

At the end of the day, technology has to be used to make a way that smoothens the human's life. This project has been designed to make registration flexible and the patients also are notified their turn of meeting the doctor.

\section{REFERENCES}

[1] Muhammad Ali Mazidi and Janice Gillispe Mazidi The 8051 microcontroller and embedded systems (India, Pearson education ltd.) (2007)

[2] www.atmel.com, AVR Microcontrollers

[3] www.dharmanitech.com_SD Card interfacing

\section{Authors}

Dr. C. Kavitha, Associate Professor and Head, Department of Electronics/ Physics, GITAM Institute of Science, GITAM University has more than 16 years of service in the academic line. She completed her PhD from Andhra University. Her qualifications are M.Sc (tech), M.Tech, M.Phil and Ph.D. She has published research articles in National and International journals. She also published general articles in daily magazines like The Hindu and Eenadu and guiding $3 \mathrm{PhD}$ candidates.

A.Venkat Ramana, having 7 years of experience in embedded systems development and WIFI testing, working as Technical Lead at Gain Span Systems India Pvt. Ltd. He completed his M.Sc. in Electronics and pursuing his $\mathrm{PhD}$ from GITAM University. He has published research articles in National and International journals.

Ms. S.Sushma Raj, Assistant Professor, Department of English, GITAM Institute of Science, GITAM University, has more than 8 years of service in the academic line. She did her M.Phil on Afro-American Literature. Her qualifications are M.A., M.Phil. She is a guest faculty for various reputed organizations. She is pursuing her $\mathrm{PhD}$ from Andhra University. She has published research articles in National and International journals. 